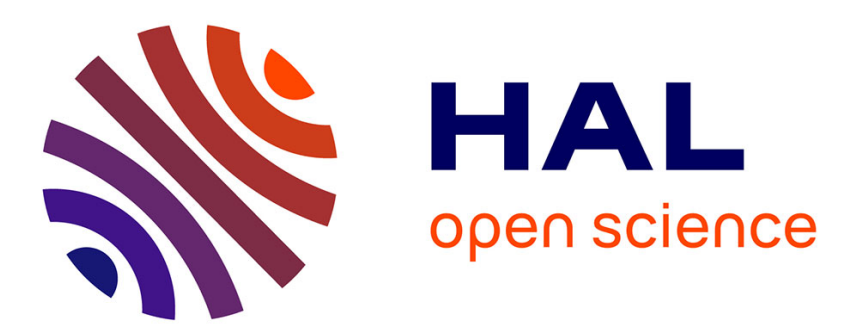

\title{
A Kalman filter for linear continuous-discrete systems with asynchronous measurements
}

\author{
Aïda Feddaoui, Nicolas Boizot, Eric Busvelle, Vincent Hugel
}

\section{To cite this version:}

Aïda Feddaoui, Nicolas Boizot, Eric Busvelle, Vincent Hugel. A Kalman filter for linear continuousdiscrete systems with asynchronous measurements. 2017 IEEE 56th Annual Conference on Decision and Control (CDC), Dec 2017, Melbourne, Australia. pp.2813-2818, 10.1109/CDC.2017.8264068 . hal-01701832

\section{HAL Id: hal-01701832 \\ https://hal.science/hal-01701832}

Submitted on 7 Feb 2019

HAL is a multi-disciplinary open access archive for the deposit and dissemination of scientific research documents, whether they are published or not. The documents may come from teaching and research institutions in France or abroad, or from public or private research centers.
L'archive ouverte pluridisciplinaire HAL, est destinée au dépôt et à la diffusion de documents scientifiques de niveau recherche, publiés ou non, émanant des établissements d'enseignement et de recherche français ou étrangers, des laboratoires publics ou privés. 


\title{
A Kalman Filter for Linear Continuous-discrete Systems with Asynchronous Measurements
}

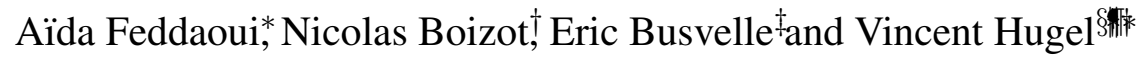

February 7, 2019

\begin{abstract}
This paper investigates an adaptation of the Kalman filter for linear continuousdiscrete system with multi-rate sampled outputs. The contribution of this article is twofold. First, we prove the exponential convergence of this observer through the existence of bounds for the Riccati matrix. Second, we highlight a technical point that allows us, under certain conditions on the sampling procedure, to prove the stability of the Riccati equation, and show that observability is preserved under multi-rate sampling. An example with two sensor outputs is given for illustration. This study can lead to applications in mobile robotics where sensors produce outputs at different rates and asynchronously.
\end{abstract}

\section{INTRODUCTION}

This paper focuses on observers for linear sampled-data systems, i.e. control systems having continuous state dynamics and a discrete measurement procedure — see e.g. [12]. In particular, we address the case where only parts of the output vector is available at a given sample time. This situation arises when the output vector is obtained through several sensors that do not have the same (possibly non-uniform) sampling rate. Such

*aida-feddaoui@etud.univ-tln.fr

†boizot@univ-tln.fr

‡busvelle@univ-tln.fr

$\S$ vincent.hugel@univ-tln.fr

`A. Feddaoui is with Laboratoire des Sciences de l'Information et des Systmes, UMR 7296, and also with Laboratoire COnception de Systmes Mcaniques et Robotiques, Universit de Toulon, 83957 La Garde, France. aida-feddaoui@etud.univ-tln.fr

${ }$ N. Boizot is with Laboratoire des Sciences de l'Information et des Systmes, UMR 7296, Universit de Toulon, 83957 La Garde, France. nicolas . boizot@univ-tln. fr

**E. Busvelle is with Laboratoire des Sciences de l'Information et des Systmes, UMR 7296, Universit de Toulon, 83957 La Garde, France. busvel le@univ-tln. fr

${ }^{\dagger}$ V. Hugel is with Laboratoire COnception de Systmes Mcaniques et Robotiques, Universit de Toulon, 83957 La Garde, France. vincent. hugel@univ-tln. fr 
systems, also known as multi-rate sampled-data systems, or asynchronous continuousdiscrete systems are often met in practice, for instance in global positioning problems, as in [15], or in the field of drone control [7]. Likewise, one can be confronted with such asynchronous systems in the field of submarine robotics, as can be seen from [2], [14] and [3].

Since we are considering systems having a non-uniform, multi-rate measurement procedure, and possibly a time-varying behaviour, the continuous-discrete framework and the Kalman filter appear as an adequate tool. Indeed, the correction gain of the Kalman filter is constantly updated, which appears preferable to observers having a fixed, offline computed, correction gain. Using a Kalman filter to perform such a task might not appear as a new idea, as it can be seen in e.g. [7, 10, 15]. However, the present paper's contribution lies in the fact that we propose a mathematical framework that allows us to state the problem - and thus write the observer's equations - and actually prove its convergence in the deterministic setting.

The main difficulties are, on the one hand, dealing with several subdivisions of time in order to represent the asynchronous outputs, and on the other hand, proving that the observer's Riccati equation is bounded over time. This latter issue is handled by following the ideas developed in [4], where only the synchronous setting is considered. Moreover, the present work is done with the purpose of expanding the results to the nonlinear setting (see e.g. $[9,1])$ and high-gain formalism (e.g. $[11,5,6])$.

Two recent articles are also worth mentioning, in which a similar task is performed, in the linear framework for [16], and in the nonlinear, high-gain setting for [18]. There are two main differences between the present approach and those two works. First, they consider observers having a fixed, off-line computed, correction gain, or in other word, Luenberger-like observers. Second, the two proposed observers are set in the pure continuous setting, whereas we consider a continuous-discrete setting where the correction step is performed only when measurements are available.

The remaining of the article is divided into four parts. Section 2 presents the control system under consideration and the corresponding Kalman filter. Section 3 deals with the proof of the exponential convergence of this observer. This demonstration heavily relies on the existence of bounds for the Riccati matrix. The proof of this result is quite technical and long, and is therefore not detailed in this article. It basically follows the ideas developed in [4], with an increased complexity coming from the asynchronicity of the measurements. However, this paper adresses the important issue of the preservation of observability under multi-rate sampling, which is a necessary condition in order to bound the Riccati matrix. An academic illustrative example is given in Section 4, and Section 5 concludes the article.

\section{DEFINITION OF THE MULTI-RATE KALMAN FIL- TER}

Throughout this article, we define a time subdivision $\left\{t_{k}\right\}_{k \in \mathbb{N}}$ as a strictly increasing sequence of real numbers with $t_{0}=0$ and $t_{k} \rightarrow \infty$ when $k \rightarrow \infty$. We denote $I d$ the identity matrix with appropriate dimensions, $|$.$| is the cardinal of a set, and \mathscr{M}_{n \times m}$ is 
the set of $(n \times m)$ matrices.

\subsection{System Under Consideration}

Let

$$
\left\{\begin{array}{l}
\dot{x}(\tau)=A(\tau) x(\tau)+B(\tau) u(\tau), \quad \text { with } x(0)=x_{0} \\
y(\tau)=C(\tau) x(\tau)
\end{array}\right.
$$

be a linear continuous system where $\tau \in \mathbb{R}^{+}, x(\tau) \in \mathbb{R}^{n}, y(\tau) \in \mathbb{R}^{n y}, u(\tau) \in \mathbb{R}^{n u}$ and $A \in \mathscr{M}_{n \times n}, B \in \mathscr{M}_{n \times n u}, C \in \mathscr{M}_{n y \times n}$. We assume that $\left(\Sigma_{c}\right)$ is observable in a classic sense (see e.g. [20]), that the elements of $A(\tau), B(\tau)$ and $C(\tau)$ are in $L^{\infty}\left(\mathbb{R}^{+}, \mathbb{R}\right)$ and are uniformly bounded by a constant $\tilde{B}>0$. Moreover, we suppose that all the elements of $C(\tau)$ are derivable at least once and have their derivative bounded over time ${ }^{1}$.

To this plant, we associate the following linear continuous-discrete system with asynchronous measurements

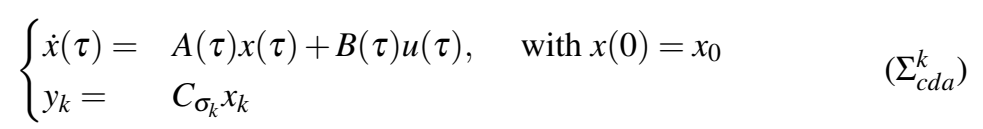

where,

- to each element of $y(\tau)$, denoted $y^{2}(i)(\tau)$ for $i \in\{1, \ldots, n y\}$, we associate a time subdivision $\left\{s_{k}^{(i)}\right\}_{k \in \mathbb{N}}$

- the subdivision of time $\left\{\tau_{k}\right\}_{k \in \mathbb{N}}$ is defined by

$$
\left\{\tau_{k}\right\}_{k \in \mathbb{N}}:=\bigcup_{i=1}^{n y}\left\{s_{k}^{(i)}\right\}_{k \in \mathbb{N}}
$$

where elements belonging to several subdivisions $\left\{s_{k}^{(i)}\right\}_{k \in \mathbb{N}}$ appear only once;

- $\sigma_{k}$ is a set, and $l_{k}^{(i)}$ is an index defined as follows

$$
\sigma_{k}=\left\{i \in\{1, \ldots, n y\} \mid \exists l_{k}^{(i)} \in \mathbb{N} \text { such that } s_{l_{k}^{(i)}}^{(i)}=\tau_{k}\right\} ;
$$

- $x_{k}=x\left(\tau_{k}\right)$ and $y_{k}$ is defined with the help of the matrix $C_{\sigma_{k}}=\left(\begin{array}{c}\vdots \\ C_{k}^{(i)} \\ \vdots\end{array}\right)_{i \in \sigma_{k}}$, where

$C_{k}^{(i)}$ is the $i^{t h}$ row of $C\left(\tau_{k}\right)$, and therefore $y_{k}^{(i)}:=C_{k}^{(i)} x_{k}$. In other words, $C_{\sigma_{k}}$ is made of the rows of $C\left(\tau_{k}\right)$ corresponding to the outputs measured at time $\tau_{k}$, and $y_{k} \in \mathbb{R}^{\left|\sigma_{k}\right|}$.

\footnotetext{
${ }^{1}$ This condition may appear restrictive but is necessary in order to apply Lemma (5) to a time-varying matrix $C$.

${ }^{2}$ This notation is not ambiguous since we only use order 1 derivatives.
} 


\subsection{Kalman Filter}

The continuous-discrete asynchronous Kalman filter is defined in two parts for all $k \in$ $\mathbb{N}$ :

1. two prediction equations defined for $\tau \in\left[\tau_{k-1}, \tau_{k}\left[\right.\right.$, with initial values $\hat{x}_{k-1}(+)$ and $S_{k-1}(+)$,

2. two correction equations at time $\tau_{k}$.

We denote by

- $\hat{x}(t)$ the estimated state for all $\tau \in\left[\tau_{k-1}, \tau_{k}[\right.$,

- $\hat{x}_{k}(-)$ the estimated state at the end of a prediction step and before a correction step,

- $\hat{x}_{k}(+)$ is the estimated state after a correction step. Note that $\hat{x}_{k}(+)$ is also the initial estimated state of a new prediction interval $\left[\tau_{k}, \tau_{k+1}[\right.$.

\section{Prediction equations}

$$
\left\{\begin{array}{l}
\dot{\hat{x}}(\tau)=A(\tau) \hat{x}(\tau)+B(\tau) u(\tau) \\
\dot{S}(\tau)=-A(\tau)^{\prime} S(\tau)-S(\tau) A(\tau)-(S Q S)(\tau)
\end{array}\right.
$$

\section{Correction equations}

$$
\left\{\begin{array}{l}
\hat{x}_{k}(+)=\hat{x}_{k}(-) \\
\quad-S_{k}(+)^{-1} \sum_{i \in \sigma_{k}} C_{k}^{(i)^{\prime}} R_{k}^{(i)^{-1}}\left(C_{k}^{(i)} \hat{x}_{k}(-)-y_{k}^{(i)}\right)\left(s_{l_{k}^{(i)}}^{(i)}-s_{l_{k}^{(i)}-1}^{(i)}\right) \\
S_{k}(+)=S_{k}(-)+\sum_{i \in \sigma_{k}} C_{k}^{(i)^{\prime}} R_{k}^{(i)-1} C_{k}^{(i)}\left(s_{l_{k}^{(i)}}^{(i)}-s_{l_{k}^{(i)}-1}^{(i)}\right)
\end{array}\right.
$$

In other words, the corrections are made with respect to each output $y_{k}^{(i)}$ actually available at time $\tau_{k}$ associated to a weighting factor equal to the time elapsed since the last time this specific output was measured.

We assume that $Q$ and $R$ are symmetric positive definite matrices, in particular $R$ is a diagonal matrix ${ }^{3}$, and both are in compact subsets of the form:

$$
\begin{gathered}
\underline{q} \text { Id } \leq Q \leq \bar{q} \text { Id }, \quad \text { with } 0<\underline{q}<\bar{q} \\
\underline{r}_{i} \leq R_{k}^{(i)} \leq \bar{r}_{i}, \quad \text { with } 0<\underline{r}_{i}<\bar{r}_{i}
\end{gathered}
$$

with $R_{k}^{(i)}$ being the $i^{\text {th }}$ diagonal component of $R$. Furthermore, we assume as well that $S(0)$ is a symmetric positive definite matrix chosen inside a compact subset.

Remark 1 Let us note that this formulation is based on the classic continuous-discrete framework (see e.g. $[8,17,13]$ ). It is also particularly adapted to the problem here addressed.

\footnotetext{
${ }^{3}$ In fact, this hypothesis can be relaxed when several outputs come from the same sensor. This topic is raised in the conclusion.
} 


\section{PROOFS}

The proof of convergence of this observer is quite immediate after proving Lemma 2 below, which gives positive lower and upper bounds for the matrix $S(\tau)$. It is actually close to the Kalman filter's convergence proof for continuous-discrete systems with synchronous outputs, which can be found in [5].

For the present case, the main difficulties are, on the one hand, to properly define the system under consideration and to deal with two types of time subdivisions : $\left\{s_{k}^{(i)}\right\}_{k \in \mathbb{N}}$ for each output $y^{(i)}, i \in\{1, \ldots, n y\}$ and $\left\{\tau_{k}\right\}_{k \in \mathbb{N}}$. On the other hand, we have to demonstrate the following lemma:

Lemma 2 Consider the prediction-correction equations $\left(K_{1}\right)$ and $\left(K_{2}\right)$ with the set of assumptions on $Q, R, S(0), A(\tau)$ and $C(\tau)$ given above. Then, there exist constants $\mu_{i}>0, i \in\{1, \ldots, n y\}$, and two scalars $0<\alpha<\beta$, such that, for all subdivisions with $\left(s_{k}^{(i)}-s_{k-1}^{(i)}\right) \leq \mu_{i}, k \in \mathbb{N}$,

$$
\alpha I d \leq S(\tau) \leq \beta I d
$$

for all $\tau \in \mathbb{R}^{+}$, before or after a correction step.

Here, $\alpha$ and $\beta$ are independent from the chosen subdivisions. Also, the constants $\mu_{i}$ are independent from the initial conditions.

\subsection{Convergence of the Kalman Filter}

We will prove here that the estimated state $\hat{x}(\tau)$ calculated by the Kalman filter $\left(K_{1}\right)$ $\left(K_{2}\right)$ converges to $x(\tau)$, the state of $\left(\Sigma_{c d a}^{k}\right)$. To this end, let the error signal be defined as $\varepsilon(\tau)=(\hat{x}-x)(\tau)$, and consider the Lyapunov function $V(\varepsilon)=\left(\varepsilon^{\prime} S \varepsilon\right)(\tau)$.

We calculate:

$$
\begin{aligned}
\dot{\varepsilon}\left(\tau_{k}\right)= & (\dot{\hat{x}}-\dot{x})(\tau)=A(\tau) \varepsilon(\tau) \\
\varepsilon_{k}(+)= & \hat{x}_{k}(+)-x\left(\tau_{k}\right) \\
= & \hat{x}_{k}(-)-x\left(\tau_{k}\right) \\
- & S_{k}(+)^{-1} \sum_{i \in \sigma_{k}} C_{k}^{(i)^{\prime}} R_{k}^{(i)}{ }^{-1}\left(C_{k}^{(i)} \hat{x}_{k}(-)-y_{k}^{(i)}\right)\left(s_{l_{k}^{(i)}}^{(i)}-s_{l_{k}^{(i)}-1}^{(i)}\right) \\
= & \varepsilon_{k}(-) \\
& -S_{k}(+)^{-1} \sum_{i \in \sigma_{k}} C_{k}^{(i))^{\prime}} R_{k}^{(i)}-1 C_{k}^{(i)} \varepsilon_{k}(-)\left(s_{l_{k}^{(i)}}^{(i)}-s_{l_{k}^{(i)}-1}^{(i)}\right)
\end{aligned}
$$

This last equation can be rewritten in a more compact form with the help of the following notations. First, let $I_{\sigma_{k}}$ be the following diagonal matrix — with $m_{k}=\left|\sigma_{k}\right|$ :

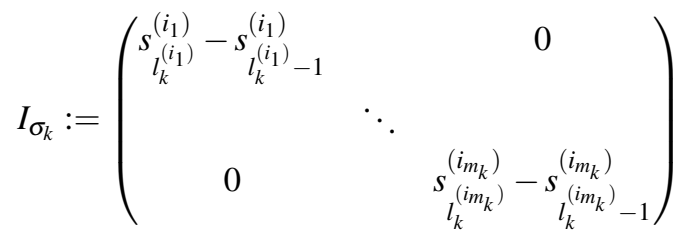


Second, the matrix $R_{\sigma_{k}}$ is defined the same way as $C_{\sigma_{k}}$, and we denote $\bar{R}_{\sigma_{k}}^{-1}:=R_{\sigma_{k}}^{-1} I_{\sigma_{k}}$. This latter matrix is well defined since $I_{\sigma_{k}}$ is clearly invertible from the definition of a time subdivision. The above equation thus can be rewritten:

$$
\varepsilon_{k}(+)=\varepsilon_{k}(-)-S_{k}(+)^{-1} C_{\sigma_{k}}^{\prime} \bar{R}_{\sigma_{k}}^{-1} C_{\sigma_{k}} \varepsilon_{k}(-) \text {. }
$$

Now, we need to compute the derivative of $V(\varepsilon)$ over time, and also the expression of $V(\varepsilon)$ after a correction step.

For $\tau \in\left[\tau_{k-1}, \tau_{k}[:\right.$

$$
\begin{aligned}
\frac{d}{d \tau} V(\varepsilon) & =\frac{d \varepsilon^{\prime} S \varepsilon}{d \tau} \\
& =\dot{\varepsilon}^{\prime} S \varepsilon+\varepsilon^{\prime}(\dot{S} \varepsilon+S \dot{\varepsilon}) \\
& =\varepsilon^{\prime} A^{\prime} S \varepsilon+\varepsilon^{\prime}\left(-A^{\prime} S-S A-S Q S\right)+\varepsilon^{\prime} S A \varepsilon \\
& =-\varepsilon^{\prime} S Q S \varepsilon
\end{aligned}
$$

At time $\tau_{k}$, we follow the ideas of [13]:

$$
\begin{aligned}
\left(\varepsilon^{\prime} S \varepsilon\right)_{k}(+) & =\varepsilon_{k}^{\prime}(-)\left[S_{k}(+)-2 C_{\sigma_{k}}^{\prime} \bar{R}_{\sigma_{k}}^{-1} C_{\sigma_{k}}\right. \\
& \left.+C_{\sigma_{k}}^{\prime} \bar{R}_{\sigma_{k}}^{-1} C_{\sigma_{k}} S_{k}(+)^{-1} C_{\sigma_{k}}^{\prime} \bar{R}_{\sigma_{k}}^{-1} C_{\sigma_{k}}\right] \varepsilon_{k}(-) \\
& =\varepsilon_{k}^{\prime}(-)\left[S_{k}(-)^{-1} S_{k}(+) S_{k}(-)^{-1}\right]^{-1} \varepsilon_{k}(-)
\end{aligned}
$$

where the transition between (3) and (4) is due to $\left(K_{2}\right)$ rewritten as:

$$
S_{k}(+)-S_{k}(-)=C_{\sigma_{k}}^{\prime} \bar{R}_{\sigma_{k}}^{-1} C_{\sigma_{k}}
$$

Still using $\left(K_{2}\right)$

$$
S_{k}(-)^{-1} S_{k}(+) S_{k}(-)^{-1}=S_{k}(-)^{-1}+S_{k}(-)^{-1} C_{\sigma_{k}}^{\prime} \bar{R}_{\sigma_{k}}^{-1} C_{\sigma_{k}} S_{k}(-)^{-1} .
$$

The matrix inversion lemma leads to

$$
\begin{aligned}
& {\left[S_{k}(-)^{-1} S_{k}(+) S_{k}(-)^{-1}\right]^{-1}} \\
& =\left[S_{k}(-)^{-1}+S_{k}(-)^{-1} C_{\sigma_{k}}^{\prime} \bar{R}_{\sigma_{k}}^{-1} C_{\sigma_{k}} S_{k}(-)^{-1}\right]^{-1} \\
& =S_{k}(-)-C_{\sigma_{k}}^{\prime}\left(\bar{R}_{\sigma_{k}}+C_{\sigma_{k}} S_{k}(-)^{-1} C_{\sigma_{k}}^{\prime}\right)^{-1} C_{\sigma_{k}}
\end{aligned}
$$

Thus

$$
\begin{cases}\left(\varepsilon^{\prime} S \varepsilon\right)_{k}(+) & =\left(\varepsilon^{\prime} S \varepsilon\right)_{k}(-) \\ & -\varepsilon_{k}^{\prime}(-) C_{\sigma_{k}}^{\prime}\left(\bar{R}_{\sigma_{k}}+C_{\sigma_{k}} S_{k}(-)^{-1} C_{\sigma_{k}}^{\prime}\right)^{-1} C_{\sigma_{k}} \varepsilon_{k}(-) \\ \frac{d \varepsilon^{\prime} S \varepsilon}{d \tau}(\tau) & =-\left(\varepsilon^{\prime} S Q S \varepsilon\right)(\tau)\end{cases}
$$

At time $\tau_{k}$, the second term of the right hand side expression of (5) is semi-positive definite, since $S(\tau)$ is positive definite for all $\tau \in \mathbb{R}^{+}$by Lemma 2 . Then

$$
\left(\varepsilon^{\prime} S \varepsilon\right)_{k}(+) \leq\left(\varepsilon^{\prime} S \varepsilon\right)_{k}(-)
$$


Since $\frac{d V}{d \tau}<0$ is decreasing over $\left[\tau_{k-1}, \tau_{k}[\right.$ for all $k \in \mathbb{N}$, and also after a discrete step, we conclude that $\frac{d V}{d \tau}<0$ for all $\tau \in \mathbb{R}^{+}$. As a consequence, $\varepsilon=(\hat{x}-x)(\tau)=0$ is an asymptotically stable point.

Moreover, this convergence is exponential. Indeed, with the help of Lemma 2:

$$
\begin{aligned}
\frac{d \varepsilon^{\prime} S \varepsilon}{d \tau}(\tau) & =-\left(\varepsilon^{\prime} S Q S \varepsilon\right)(\tau) \\
& \leq-\alpha \bar{q}\left(\varepsilon^{\prime} S \varepsilon\right)(\tau) \\
\varepsilon^{\prime} S \varepsilon(\tau) & \leq\left(\varepsilon^{\prime} S \varepsilon\right)(0) e^{-\alpha \bar{q} \tau} \\
\|\varepsilon(\tau)\|^{2} & \leq \frac{\beta}{\alpha}\|\varepsilon(0)\|^{2} e^{-\alpha \bar{q} \tau} .
\end{aligned}
$$

\subsection{Stability of the Riccati Equation}

The present section is dedicated to the proof of Lemma 2. It follows the ideas exposed in [4] for synchronous continuous-discrete systems, and expands them to the multi-rate setting. For the sake of brevity, this quite long proof is not reproduced here. We only sketch the main ideas, and discuss the main technical differences between the classic continuous-discrete case and the multi-rate one.

As it is done in [4] —but also in [11] for continuous systems - the existence of bounds for the Riccati equation is demonstrated in two parts:

1. the proof of the existence of an upper bound;

2. the proof of the existence of a lower bound.

Each part, consists of first exhibiting a bound in small-time, and second, finding a long-time bound.

The small-time bounds are easily obtained with the help of Gronwall's lemma. However, they become useless as time increases, since they come from an exponential term that increases with time.

The argument used to prove the existence of a long-time bound varies depending on wether the upper bound or lower bound is considered.

In the first case, the key point is the regularity of the Riccati equation. It requires a limiting condition on the maximum step size of time subdivisions. However, at this stage, this condition only applies to the subdivision $\left\{\tau_{k}\right\}_{k \in \mathbb{N}}$.

In the second case, the long-time lower bound depends on whether or not observability is preserved under multi-rate sampling. For this purpose, we prove that the Gramm matrix of $\left(\Sigma_{c d a}^{k}\right)$ is always positive definite, provided that each subdivision $\left\{s_{k}^{(i)}\right\}_{k \in \mathbb{N}}, i \in\{1, \ldots, n y\}$, has a small enough maximum step size.

In the remaining part of this section, we discuss the following key point mentioned in the previous paragraph:

Let $G_{c d a}(T)$ denote the Gramm matrix of the system $\left(\Sigma_{c d a}^{k}\right)$, associated to the fixed time $T>0$. 
If all the subdivisions $\left\{s_{k}^{(i)}\right\}_{k \in \mathbb{N}}, i \in\{1, \ldots, n y\}$, have a small enough maximum step size, then there exists $a>0$ such that aId $<G_{c d a}(T)$.

1. First of all, the Gramm matrix at time $T, G_{c d a}(T)$ is defined as

$$
\begin{aligned}
G_{c d a}(T) & =\sum_{i=1}^{n y} \sum_{j=1}^{\lambda_{k}^{(i)}} \varphi_{a}^{\prime}\left(s_{j}^{(i)}, T\right) C_{j}^{(i)^{\prime}} C_{j}^{(i)} \varphi_{a}\left(s_{j}^{(i)}, T\right)\left(s_{j}^{(i)}-s_{j-1}^{(i)}\right) \\
& =\sum_{i=1}^{n y} G_{c d a}^{(i)}(T)
\end{aligned}
$$

where

- the index $\lambda_{k}^{(i)}$ is such that:

$$
\lambda_{k}^{(i)}=\max \left\{l \in \mathbb{N} \text { such that } s_{l}^{(i)} \leq T\right\}
$$

- $\varphi_{a}(t, s)$ is the resolvent of $\left(\Sigma_{c}\right)$, with $\varphi_{a}(s, s)=I d$.

2. Second, let us state the following lemma about continuous systems.

Lemma 3 ([11], Ch. 6) Let $G_{c}(T)$ be the continuous Grammian of $\left(\Sigma_{c}\right)$ at time $T$ :

$$
G_{c}(T)=\int_{0}^{T} \varphi_{a}^{\prime}(v, T) C^{\prime}(v) C(v) \varphi_{a}(v, T) d v .
$$

If the elements of $A(\tau)$ and $C(\tau)$ are uniformly bounded in $L^{\infty}\left(\mathbb{R}^{+}, \mathbb{R}\right)$ by some constant $B>0$, then there exist two positive scalars $0<\bar{\alpha}<\bar{\beta}$, that depend on $B$ and $T$ only, such that

$$
\bar{\alpha} I d \leq G_{c}(T) \leq \bar{\beta} I d
$$

Remark 4 Notice that by the definition of the matrix product,

$$
\begin{aligned}
G_{c}(T) & =\sum_{i=1}^{n y} G_{c}^{(i)}(T) \\
& =\sum_{j=1}^{n y} \int_{0}^{T} \varphi_{a}^{\prime}(v, T) C^{(i)^{\prime}}(v) C^{(i)}(v) \varphi_{a}(v, T) d v
\end{aligned}
$$

In order to use this lemma, let us write:

$$
G_{c}(T)=G_{c}(T)-G_{c d a}(T)+G_{c d a}(T)
$$

Now, we want to combine Lemma 3 with the fact that $G_{c}(T)-G_{c d a}(T)$ can be made small enough. This is done with the help of the following lemma, which can be proved using similar arguments as the proof of Lemma 3.11 in [4]. 
Lemma 5 Let $m(t), t \in[0, T]$, be a $(n \times n)$ symmetric matrix, at least differentiable once.

Let $\mu$ be a positive constant, and $\left\{t_{k}\right\}_{k \in\{0,1, \ldots, k\}}$ an arbitrary finite subdivision of $[0, T]$, such that $t_{j}-t_{j-1} \leq \mu$ for all $j \in \mathbb{N}$, with $t_{0}=0$ and $t_{k}$, the maximal element of this subdivision, is such that $T-t_{k} \leq \mu$.

We suppose that all the coefficients of $m$ have their derivative bounded over time.

Then

$$
\int_{0}^{T} m(v) d v-\sum_{j=1}^{k} m\left(t_{j}\right)\left(t_{j}-t_{j-1}\right) \leq \mu(K T+L) I d,
$$

where $L=\sup \|m(t)\|_{2}$, with $\|\cdot\|_{2}$ the matrix norm induced by the euclidean norm and $K=\frac{n}{2} \max _{k, l, t}^{t}\left(\left|m_{k, l}^{\prime}(t)\right|\right)$, with $m_{k, l}^{\prime}(t)$ the element of the $k^{\text {th }}$ row and $l^{\text {th }}$ column of the matrix $m^{\prime}(t)$.

3. By lemma 5, there are constants $L>0$ and $K_{i}>0$ such that for all $i \in\{1, \ldots, n y\}$ , we have at time $T$ :

$$
\begin{aligned}
& G_{c}^{(i)}(T)-G_{c d a}^{(i)}(T) \\
= & \int_{0}^{T} \varphi_{a}^{\prime}(v, T) C^{(i)^{\prime}}(v) C^{(i)}(v) \varphi_{a}(v, T) d v \\
& \lambda_{k}^{(i)} \varphi_{a}^{\prime}\left(s_{j}^{(i)}, T\right) C_{j}^{(i)} C_{j}^{(i)} \varphi_{a}\left(s_{j}^{(i)}, T\right)\left(s_{j}^{(i)}-s_{j-1}^{(i)}\right) \\
\leq & \mu_{i}\left(K_{i} T+L\right) I d
\end{aligned}
$$

Let us apply Lemma 3 on $G_{c}(T)$, using the expression (5) and the inequality (6) :

$$
\begin{aligned}
\alpha I d & \leq G_{c}(T) \\
& =\sum_{i=1}^{n y} G_{c}^{(i)}(T)-\sum_{i=1}^{n y} G_{c d a}^{(i)}(T)+\sum_{i=1}^{n y} G_{c d a}^{(i)}(T) \\
& \leq \sum_{i=1}^{n y} G_{c d a}^{(i)}(T)+\sum_{i=1}^{n y} \mu_{i}\left(K_{i} T+L\right) I d
\end{aligned}
$$

It allows us to conclude that:

$$
\left[\bar{\alpha}-\sum_{i=1}^{n_{y}} \mu_{i}\left(K_{i} T+L\right)\right] I d \leq G_{c d a}(T) .
$$

Finally, by choosing each $\mu_{i}, i \in\{1, \ldots, n y\}$ sufficiently small to make $\left(\bar{\alpha}-\sum_{i=1}^{n_{y}} \mu_{i}\left(K_{i} T+L\right)\right)$ positive, then, the linear continuous-discrete system with multi-rate outputs $\left(\Sigma_{c d a}^{k}\right)$ associated to $\left(\Sigma_{c}\right)$ is observable as soon as it is the case for $\left(\Sigma_{c}\right)$. 


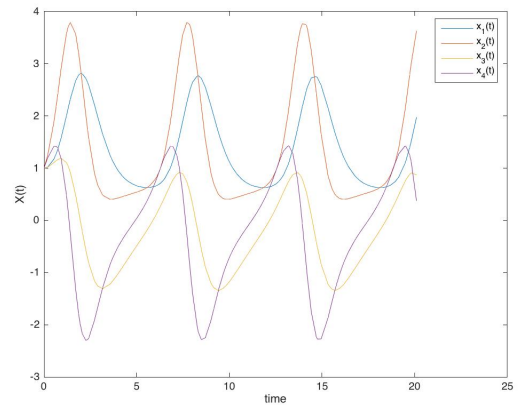

Figure 1: For proper display purposes, the other graphs are shown on smaller time windows.

Remark 6 One may think that the definition of the Kalman filter $\left(K_{1}\right)-\left(K_{2}\right)$ proposed in this article could be written in more simple terms, especially the sum depending on the subdivisions $\left\{s_{k}^{(i)}\right\}_{k \in \mathbb{N}}, i \in\{1, \ldots, n y\}$ in $\left(K_{2}\right)$. It is unfortunately not the case. Actually, considering the subdivision $\left\{\tau_{k}\right\}_{k \in \mathbb{N}}$ instead of $\left\{s_{k}^{(i)}\right\}_{k \in \mathbb{N}}$ leads, when computing iteratively $S_{k}(+)$, either to overlapping intervals of time or disjointed intervals of time.

\section{ILLUSTRATIVE EXAMPLE}

The behaviour of the Kalman filter defined in $\left(K_{1}\right)-\left(K_{2}\right)$ is illustrated with the following autonomous time-varying system, where $x(\tau) \in \mathbb{R}^{4}$ :

$$
\begin{aligned}
\dot{x}(\tau) & =\left(\begin{array}{cccc}
-1 & 1 & 0 & 0 \\
0 & \cos (t) & 0 & \sin (t) \\
0 & 0 & -1 & 1 \\
0 & -\sin (t) & 0 & \cos (t)
\end{array}\right) x(\tau) \\
& =A(\tau) x(\tau) .
\end{aligned}
$$

This system is an academic, time-varying, constantly oscillating system, as it is illustrated in Figure 1. For simplicity, we do not consider control inputs since, as it is well known from linear systems control theory, they do not play a critical role.

The output vector $y(\tau) \in \mathbb{R}^{2}$ is given by:

$$
\begin{aligned}
y(\tau) & =\left(\begin{array}{llll}
1 & 0 & 0 & 0 \\
0 & 0 & 1 & 0
\end{array}\right) x(\tau) \\
& =C x(\tau) .
\end{aligned}
$$

According to $[20,19]$ it is immediate to check that the continuous system made of (7)(8) is observable ${ }^{4}$. However, observability is lost as soon as $C(\tau)$ is restricted to only one of its two lines.

\footnotetext{
${ }^{4}$ In particular, following the notations of [19], it's observability index equals 2 .
} 


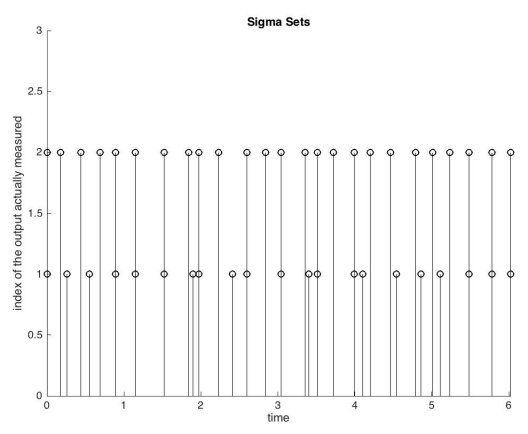

Figure 2: A dot represents a measured output. For the run here displayed, the maximum step sizes are, 0.48 for $\left\{s_{k}^{(1)}\right\}, 0.37$ for $\left\{s_{k}^{(2)}\right\}$ and 0.37 for $\left\{\tau_{k}\right\}$.

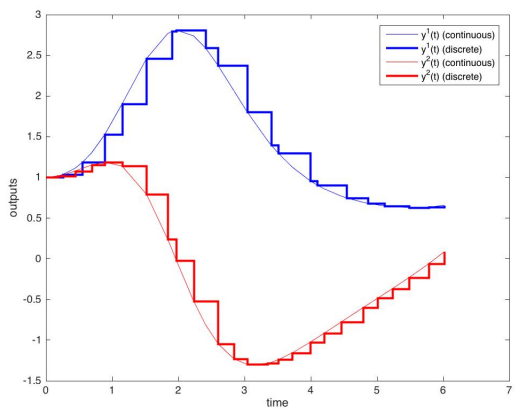

Figure 3: Measured outputs (continuous outputs are shown for information).

In this example, the two outputs $y^{(1)}(\tau)=C^{(1)} x(\tau)$ and $y^{(2)}(\tau)=C^{(2)} x(\tau)$ are measured using non-uniform subdivisions of time. Each one of those two subdivisions are obtained by randomly modifying a uniform one - starting with an initial period of 0.3 time units for $y^{(1)}(t)$, and 0.25 time units for $y^{(2)}(t)$. This is illustrated in Figure 2 where the three subdivisions $\left\{s_{k}^{(1)}\right\},\left\{s_{k}^{(2)}\right\}$ and $\left\{\tau_{k}\right\}$ together with the sets $\sigma_{k}$ clearly appear. The output trajectories are displayed in Figure 3.

We performed a simulation of the proposed observer following the procedure below.

The initial state of the system is $x(0)=(1,-1,1,-1)$. The initial state of the observer is set to $\hat{x}(0)=(-3,4,-5,6)$. The matrices $Q$ and $R$, are simply equal to identity.

In order to select a good initial value for the Riccati matrix, we first run the observer with $S(0)=I d$, while forcing the error term to 0 in the correction equations. This procedure provides a stabilised Riccati matrix, which is used as $S(0)$ to run our experiment.

Finally the results of this simple simulation are displayed in Figure 4. 


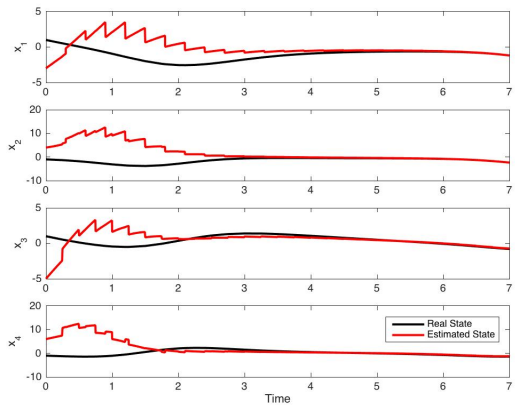

Figure 4: Convergence of the estimated state toward the real state.

\section{CONCLUSIONS AND FUTURE WORKS}

In this paper, an adaptation of the Kalman filter to continuous-discrete systems with multi-rate sampled outputs has been proposed. To this end, each correction step involves a weighted sum of the output errors calculated on the basis of the measurements available at this sample time. Moreover, we showed the observability of $\left(\Sigma_{c d a}^{k}\right)$ provided that $\left(\Sigma_{c}\right)$, the associated continuous system, is observable.

Some improvements are left for the future. To begin with, we plan to consider $n s$ the number of sensors instead of $n y$ the number of outputs. Two different outputs correspond to the same sensor if their associated subdivisions of time are the same. By doing so, larger $R^{(i)}$ sub-matrices can be considered and cross-correlations between outputs delivered by a same sensor can be taken into account.

Furthermore, the presence of redundant sensors can lead to an improved version of Lemma 2. Indeed, the maximum step size condition on the time subdivision of a given sensor could be relaxed provided there is an active redundant sensor.

\section{ACKNOWLEDGMENTS}

\section{References}

[1] Vincent Andrieu, Madiha Nadri, Ulysse Serres, and Jean-Claude Vivalda. Selftriggered continuous discrete observer with updated sampling period. Automatica, 62:106-113, 2015.

[2] C. Barbalata, V. De Carolis, M. W. Dunnigan, Y. Petillot, and D. Lane. An adaptive controller for au- tonomous underwater vehicles. In Proceeding og IEEE/RSJ International Conference on Intelligent Robots and Systems (IROS), pages 1658 - 1663, 2015.

[3] M. Blain, S. Lemieux, and R. Houde. Implementation of a ROV navigation system using acoustic/doppler sensors and kalman filtering. In Oceans 2003. Cel- 
ebrating the Past ... Teaming Toward the Future (IEEE Cat. No.03CH37492). Institute of Electrical and Electronics Engineers (IEEE), 2003.

[4] Nicolas Boizot and Eric Busvelle. On the stability of a differential riccati equation for continuous-discrete observers. International Journal of Control, 89(2):322336, 2015.

[5] Nicolas Boizot, Eric Busvelle, and Jean-Paul Gauthier. Adaptive-gain extended kalman filter: Extension to the continuous-discrete case. In Proceedings of the European control conference, 2009.

[6] Nicolas Boizot, Eric Busvelle, and Jean-Paul Gauthier. An adaptive high-gain observer for nonlinear systems. Automatica, 46(9):1483-1488, 2010.

[7] P.-J. Bristeau, E. Dorveaux, D. Vissière, and N. Petit. Hardware and software architecture for state estimation on an experimental low-cost small-scaled helicopter. Control Engineering Practice, 18(7):733-746, 2010.

[8] F. Deza, E. Busvelle, J-P. GAUTHIER, and D. Rakotopara. High-gain estimation for nonlinear systems. Systems and Control Letters, 18:295 - 299, 1992.

[9] Thach Ngoc Dinh, Vincent Andrieu, Madiha Nadri, and Ulysse Serres. Continuous-discrete time observer design for lipschitz systems with sampled measurements. IEEE Transactions on Automatic Control, 60(3):787-792, 2014.

[10] Cécile Durieu, Marie-José Aldon, and Dominique Meizel. Multisensory data fusion for localisation in mobile robotics. Traitement du signal, 13(2):143-166, 1996.

[11] Jean-Paul Gauthier and Ivan Kupka. Deterministic Observation Theory and Applications. Cambridge University Press, 2001. pages 104-113.

[12] Akira Ichikawa and Hitoshi Katayama. Linear Time Varying Systems and Sampled-data Systems, volume 265 of Lecture Notes in Control and Information Sciences. Springer-Verlag London, 2001.

[13] Andrew Jazwinski. Stochastic Processus and Filtered Theory (reprint). Dover Publications, 2007.

[14] Divine Maalouf, Ahmed Chemori, and Vincent Creuze. L1 adaptive depth and pitch control of an underwater vehicle with real-time experiments. Ocean Engineering, Elsevier, 98:66-77, March 2015.

[15] David Mayhew and Pushkin Kachroo. Multirate sensor fusion for gps using kalman filtering, fuzzy methods, and map matching. In Proceedings of SPIE: Mobile Robots XIII and Intelligent Transportation Systems, volume 3525(1), pages 440-449, 1998.

[16] Miad Moarref and Luis Rodrigues. Observer design for linear multi-rate sampleddata systems. In Proceedings of American Control Conference, 2014. 
[17] M. Nadri and H. Hammouri. Design of a continuous-discrete observer for state affine systems. Applied Mathematics Letters, 16:967 - 974, 2003.

[18] Yanjun Shen, Daoyuan Zhang, and Xiaohua Xia. Continuous observer design for a class of multi-output nonlinear systems with multi-rate sampled and delayed output measurements. Automatica, 75:127-132, 2017.

[19] L. S. Shieh, S. Ganesan, and J. M. Navarro. Transformations of a class of time varying multivariable control systems to block companion forms. Comput. Math. Applic., 14(6):471-477, 1987.

[20] Eduardo Sontag. Mathematical Control Theory, volume 6 of Texts in Applied Mathematics. Springer-Verlag New-York Berlin Heidelberg, 1990. pages 207208. 